BMJ Open Diabetes

Research \& Care

\section{Oral diabetes medication monotherapy and short-term mortality in individuals with type 2 diabetes and coronary artery disease}

\author{
Sridharan Raghavan, ${ }^{1,2,3}$ Wenhui G Liu, ${ }^{1}$ David R Saxon, ${ }^{1,4}$ Gary K Grunwald, ${ }^{1,5}$ \\ Thomas M Maddox, ${ }^{6}$ Jane E B Reusch, ${ }^{1,4}$ Seth A Berkowitz, ${ }^{7}$ Liron Caplan ${ }^{1,8}$
}

To cite: Raghavan S, Liu WG, Saxon DR, et al. Oral diabetes medication monotherapy and short-term mortality in individuals with type 2 diabetes and coronary artery disease. BMJ Open Diab Res Care 2018;6:e000516. doi:10.1136/ bmjdrc-2018-000516

- Additional material is published online only. To view please visit the journal online (http://dx.doi.org/10.1136/10. 1136/bmjdrc-2018-000516).

Received 19 January 2018 Accepted 27 May 2018

Check for updates

For numbered affiliations see end of article.

Correspondence to Dr Sridharan Raghavan; sridharan.raghavan@ucdenver. edu

\section{ABSTRACT}

Objective To determine whether sulfonylurea use, compared with non-sulfonylurea oral diabetes medication use, was associated with 2-year mortality in individuals with well-controlled diabetes and coronary artery disease (CAD).

Research design and methods We studied 5352 US veterans with type 2 diabetes, obstructive CAD on coronary angiography, hemoglobin $\mathrm{A} 1 \mathrm{c} \leq 7.5 \%$ at the time of catheterization, and taking zero or one oral diabetes medication (categorized as no medications, non-sulfonylurea medication, or sulfonylurea). We estimated the association between medication category and 2-year mortality using inverse probability of treatment-weighted (IPW) standardized mortality differences and IPW multivariable Cox proportional hazards regression.

Results $49 \%, 35 \%$, and $16 \%$ of the participants were on no diabetes medications, non-sulfonylurea medications, and sulfonylureas, respectively. In individuals on no medications, non-sulfonylurea medications, and sulfonylureas, the unadjusted mortality rates were $6.6 \%, 5.2 \%$, and $11.9 \%$, respectively, and the IPW-standardized mortality rates were $5.9 \%, 6.5 \%$, and $9.7 \%$, respectively. The standardized absolute 2-year mortality difference between non-sulfonylurea and sulfonylurea groups was $3.2 \%(95 \% \mathrm{Cl} 0.7$ to 5.7) $(p=0.01)$. In Cox proportional hazards models, the point estimate suggested that sulfonylurea use might be associated with greater hazard of mortality than non-sulfonylurea medication use, but this finding was not statistically significant (HR $1.38(95 \% \mathrm{Cl} 1.00$ to 1.93), $p=0.05$ ). We did not observe significant mortality differences between individuals on no diabetes medications and non-sulfonylurea users.

Conclusions Sulfonylurea use was common (nearly one-third of those taking medications) and was associated with increased 2-year mortality in individuals with obstructive CAD. The significance of the association between sulfonylurea use and mortality was attenuated in fully adjusted survival models. Caution with sulfonylurea use may be warranted for patients with well-controlled diabetes and CAD, and metformin or newer diabetes medications with cardiovascular safety data could be considered as alternatives when individualizing therapy.

\section{Significance of this study}

What is already known about this subject?

- Randomized trials and comparative effectiveness studies of metformin versus sulfonylurea use for oral diabetes treatment have demonstrated conflicting results regarding their relative safety.

- There is specific concern about harms of sulfonylureas in individuals with pre-existing coronary artery disease, who may be more susceptible to direct cardiotoxic effects or hypoglycemia from sulfonylurea use.

What are the new findings?

- Sulfonylurea monotherapy was common during the study period (2005-2015) in a population of individuals with type 2 diabetes, reasonably controlled glycemia (hemoglobin A1c $\leq 58 \mathrm{mmol} / \mathrm{mol}$ ), and obstructive coronary artery disease.

- In the study population, treatment with sulfonylureas was associated with greater 2-year all-cause mortality than treatment with non-sulfonylurea medications or no diabetes medications, but the association was not statistically significant in fully adjusted survival analysis.

- There was no difference in 2-year all-cause mortality between individuals treated with non-sulfonylurea medications and those not taking a diabetes medication.

\section{How might these results change the focus of} research or clinical practice?

- In individuals with reasonably controlled type 2 diabetes and obstructive coronary artery disease, oral diabetes monotherapy with non-sulfonylurea medications (especially metformin) may be associated with lower short-term mortality than treatment with sulfonylureas.

\section{INTRODUCTION}

Individuals with type 2 diabetes mellitus and cardiovascular disease are at an elevated risk of cardiovascular events and mortality, but may also be at risk for overtreatment. ${ }^{12}$ 
While several new diabetes medications have demonstrated cardiovascular benefits, ${ }^{3-7}$ the vast majority of individuals with diabetes and lower hemoglobin A1c (HbA1c) levels are on older oral medications (eg, metformin, sulfonylureas) for which specific cardiovascular outcomes trials have not been performed. Several observational studies and randomized trials have compared metformin and sulfonylurea therapy in patients with diabetes. ${ }^{8-15}$ While most have demonstrated better outcomes with metformin treatment, the studies vary methodologically and in quality, ${ }^{8}{ }^{15}$ and sulfonylurea use remains very common. ${ }^{16} 17$ One area of uncertainty is whether improved outcomes associated with metformin compared with sulfonylurea use are attributable to the benefits of metformin or harms related to sulfonylureas. ${ }^{8}$ Individual studies, meta-analyses, and review articles arrive at conflicting conclusions, and a clear consensus has not emerged. ${ }^{8-15}$

The American Diabetes Association advocates an individualized approach to diabetes management that incorporates patient preferences, comorbidities, and diabetes complications. ${ }^{18}{ }^{19}$ However, the interactions between medication efficacy, safety, and comorbid conditions are not addressed in prospective trials. For ethical reasons, randomized trials do not compare harms of treatment as their primary objective, and they are often not powered to detect harms of treatment as secondary outcomes if drug-related adverse events occur infrequently. As such, observational studies, particularly those derived from clinical data sets, are necessary to evaluate treatment-related harms, although care must be taken to avoid bias. In addition, randomized trials are not well suited to provide evidence for individualized or stratified treatment approaches that apply to specific subgroups but not to the entire population of patients with a disease. ${ }^{20-22}$ Studies are needed that focus on specific patient populations to support an individualized approach to diabetes care. With regard to cardiovascular safety of older oral diabetes treatments, few studies have specifically examined these medications in individuals with known coronary artery disease (CAD) in whom cardiovascular harms may be more readily apparent. ${ }^{23}{ }^{24}$ In addition, prior studies have not focused on individuals with well-controlled glycemia, a patient population that may be at increased risk of hypoglycemia or overtreatment, ${ }^{2}$ for whom providers may have greater flexibility with diabetes treatment choice, and in whom minimizing harm from diabetes treatment is an important goal.

Accordingly, the aim of this study was to assess the association between sulfonylureas, non-sulfonylurea oral treatments, and no diabetes medications and shortterm mortality in individuals with objectively confirmed CAD and well-controlled diabetes. Elucidating the comparative effects of the most commonly used oral diabetes medications on short-term clinical outcomes in individuals with CAD could inform diabetes medication optimization at the time of a myocardial infarction
(MI) or percutaneous coronary intervention in individuals at high risk of short-term mortality.

\section{METHODS}

\section{Study population}

We used data from the US Veterans Affairs (VA) Clinical Assessment, Reporting, and Tracking Program (CART), a database of US veterans who have undergone cardiac catheterization since $2005 .{ }^{25}$ Patient and procedural data in CART can be merged with longitudinal VA patient data, including pharmacy data, laboratory testing, procedure codes, diagnosis codes, and vital status. For this study, we included individuals in CART with type 2 diabetes, based on a previously validated algorithm using VA data. ${ }^{26}$ Briefly, we included patients with one (from an inpatient encounter) or two (from outpatient encounters) InternationalClassification of Diseases, Ninth Revision, Clinical Modification (ICD-9-CM) diagnosis codes for type 2 diabetes occurring within a 24-month period prior to presentation for cardiac catheterization, or with a filled prescription for a diabetes medication. ${ }^{26}$

In this analysis, we focused on individuals with well-controlled type 2 diabetes using simple diabetes regimens, for whom there may be greater flexibility with medication choice and who may face a real risk of harm from certain treatments. Accordingly, we defined well-controlled glycemia as having an HbAlc $\leq 58 \mathrm{mmol} / \mathrm{mol}$ $(7.5 \%)$ within 6 months prior to catheterization, and we limited our study to those taking zero or one oral diabetes medication at the time of catheterization. As we were interested in addressing the safety and/or benefits of sulfonylureas and other diabetes medications specifically in high-risk individuals with cardiovascular disease, we included individuals who underwent catheterization for acute coronary syndrome (ST elevation MI, non-ST elevation MI, unstable angina) or who had obstructive CAD on elective angiography. Obstructive CAD was defined as any stenosis $\geq 50 \%$ in the left main coronary artery and/ or $\geq 70 \%$ in any other coronary artery.

\section{Exposure}

The primary exposure was diabetes medication category at the time of presentation with MI or for angiography (no medications, non-sulfonylurea, or sulfonylurea). We determined baseline medication category by examining prescription data over 90 days prior to the date of catheterization. Individuals with a filled prescription for either a non-sulfonylurea or sulfonylurea medication in the 90 days preceding catheterization were considered exposed, and individuals with a prescription for more than one diabetes medication within 90 days of catheterization were excluded. To be categorized as on no medications, individuals had to have no filled prescriptions for any diabetes medication in the 90 days prior to catheterization. The 90-day window for a medication prescription was chosen as this is the maximum 
duration that is typically covered or dispensed for a single prescription in the VA.

\section{Outcome}

The primary outcome was 2-year all-cause mortality based on VA vital status data. VA vital status data integrate multiple VA and non-VA data sources, including the VA Beneficiary Identification Records Locator Subsystem Death File, VA Medicare Vital Status File, and the Social Security Administration Death Master File. We focused on short-term mortality for two reasons. First, there is greater opportunity for bias in the estimated association between baseline medication exposure and outcomes as the follow-up time increases. Second, the period of time around the performance of coronary angiography represents an opportunity for diabetes treatment modification or optimization. Estimating the association between diabetes treatment at the time of CAD diagnosis and a near-term clinical outcome provides the best observational evidence for treatment modification at the time of cardiac catheterization or acute coronary syndrome.

\section{Statistical analysis}

We compared baseline characteristics between medication groups using $\chi^{2}$ tests for categorical variables and Mann-Whitney-Wilcoxon tests for continuous and ordinal variables. We plotted unadjusted Kaplan-Meier survival curves after cardiac catheterization for each medication category, and compared survival probability with the log-rank test.

We used generalized boosted regression (R V.3.1, TWANG package $)^{27}$ to estimate propensity scores for medication category based on demographics (age, sex, race), $\mathrm{CAD}$ risk factors (family history of $\mathrm{CAD}$, tobacco use, hypertension, hyperlipidemia, body mass index, Framingham cardiovascular disease risk score), comorbidities (peripheral arterial disease, congestive heart failure, chronic obstructive pulmonary disease, chronic kidney disease (CKD), dialysis, depression, post-traumatic stress disorder), angiography-related variables (indication for catheterization, postangiography revascularization), and diabetes-related variables (baseline HbAlc, diabetes duration). CKD was based on indication as a pre-existing problem on the standardized CART cardiac catheterization assessment or the presence of one inpatient or two outpatient ICD-9-CM diagnosis codes for CKD occurring within 2 years prior to the date of cardiac catheterization. Generalized boosted regression differs from standard approaches to estimate propensity scores in that it applies an iterative process with multiple regression trees to fit complex and non-linear functions between treatment and covariates to identify the propensity score model that yields the best covariate balance between treatment groups, including more than two groups as in the current study, while avoiding overfitting. ${ }^{27}$ Because site-level effects cannot be accounted for in the TWANG package for generalized boosted regression, we examined variation in treatment and mortality across VA sites as a potential source of residual confounding. To address this issue, we assessed whether mortality in our data set varied across VA sites, which did not (online supplementary material figure 1 ). In addition, we performed a sensitivity analysis in which we excluded the three VA sites (Las Vegas, Nevada; Temple, Texas; and Wilkes-Barre, Pennsylvania) where there were no individuals taking sulfonylurea medications.

We next used the estimated propensity of treatment from generalized boosted regression to generate an inverse probability of treatment-weighted (IPW) study population. We evaluated covariate balance before and after IPW using standardized differences; all covariates were adequately balanced (absolute difference $<10 \%$ ) except for CKD and dialysis. Therefore, we included CKD and dialysis as covariates in the primary analyses, and we performed a sensitivity analysis in which we excluded participants with CKD or on dialysis to achieve better confounder balance between medication categories.

We estimated the association between medication category and 2-year mortality using IPW-standardized mortality rates and absolute risk differences between medication categories. Secondarily, we used IPW multivariable Cox proportional hazards regression to estimate the association between medication category and relative hazard of mortality. All analyses were conducted in SAS V.9.4 and R V.3.1 (R Foundation for Statistical Computing, Vienna, Austria). All statistical codes are available on request.

\section{RESULTS}

Of 5352 participants, 2603 (49\%), 1889 (35\%), and $860(16 \%)$ were on no diabetes medications, non-sulfonylurea medications, and sulfonylureas, respectively. Prior to inverse probability of treatment weighting, there were statistically significant differences between medication categories across most covariates (table 1). Individuals on non-sulfonylurea medications were less likely to have congestive heart failure, CKD, or be on dialysis compared with those on no diabetes medications or taking a sulfonylurea. After IPW, covariates were well balanced between medication categories; the standardized absolute difference between medication categories was $<10 \%$ for all covariates except for CKD and dialysis (table 2).

Among those on non-sulfonylurea medications, 95\% were on metformin, $3 \%$ were on pioglitazone or rosiglitazone, and $<1 \%$ were on other medications. Among those taking a sulfonylurea, $69.2 \%$ were on glipizide, $30.2 \%$ were on glyburide, and $0.6 \%$ were on glimepiride. Participants in each treatment category tended to remain in that category over the first year after cardiac catheterization. Of the participants on no diabetes medications at baseline, $85.3 \%$ remained on no medications at 1 year after catheterization; $94.0 \%$ of those on non-sulfonylurea medication at baseline remained on monotherapy with a non-sulfonylurea medication at 1 year; and $85.0 \%$ of those treated with a sulfonylurea at baseline continued 
Table 1 Study participant characteristics

\begin{tabular}{|c|c|c|c|c|c|}
\hline & Overall, $n=5352$ & $\begin{array}{l}\text { No medications, } \\
n=2603\end{array}$ & $\begin{array}{l}\text { Non-sulfonylurea, } \\
n=1889\end{array}$ & $\begin{array}{l}\text { Sulfonylurea, } \\
\mathrm{n}=860\end{array}$ & $P$ values \\
\hline Age (years), mean (SD) & $67.1(8.0)$ & $68.8(8.8)$ & $65.5(7.2)$ & $67.8(8.1)$ & $<0.0001$ \\
\hline Male, n (\%) & 5277 (98.6) & $2568(98.7)$ & $1854(98.2)$ & $855(99.4)$ & 0.03 \\
\hline \multicolumn{6}{|l|}{ Race, n (\%) } \\
\hline White & $4473(83.6)$ & $2142(82.3)$ & $1631(86.3)$ & $700(81.4)$ & \multirow[t]{5}{*}{0.0002} \\
\hline Black & $750(14.0)$ & $395(15.2)$ & $213(11.3)$ & $142(16.5)$ & \\
\hline Asian & $32(0.6)$ & $18(0.7)$ & $13(0.7)$ & $1(0.1)$ & \\
\hline Pacific Islander & $61(1.1)$ & $24(0.9)$ & $25(1.3)$ & $12(1.4)$ & \\
\hline Native American & $36(0.7)$ & $24(0.9)$ & $7(0.4)$ & $5(0.6)$ & \\
\hline Family history of CAD, $\mathrm{n}(\%)$ & $964(18.0)$ & $449(17.3)$ & $379(20.1)$ & $136(15.8)$ & 0.01 \\
\hline Tobacco, n (\%) & $318(59.4)$ & 1559 (59.9) & $1137(60.2)$ & $484(56.3)$ & 0.1 \\
\hline Hypertension, n (\%) & 4986 (93.2) & 2403 (92.3) & 1764 (93.4) & $819(95.2)$ & 0.01 \\
\hline Hyperlipidemia, n (\%) & 4907 (91.7) & $2373(91.2)$ & $1756(93.0)$ & $778(90.5)$ & 0.04 \\
\hline BMI $\left(\mathrm{kg} / \mathrm{m}^{2}\right)$, mean (SD) & $31.2(5.5)$ & $31.2(5.9)$ & $31.6(5.5)$ & $30.9(5.4)$ & $<0.0001$ \\
\hline Framingham risk score, mean (SD) & $22.8(11.2)$ & $23.8(11.4)$ & $21.6(10.4)$ & $23.3(11.5)$ & $<0.0001$ \\
\hline PAD, n (\%) & $984(18.4)$ & $488(18.8)$ & 305 (16.2) & $191(22.2)$ & 0.0006 \\
\hline CHF, n (\%) & $739(13.8)$ & $396(15.2)$ & $173(9.2)$ & $170(19.8)$ & $<0.0001$ \\
\hline COPD, n (\%) & $930(17.4)$ & $476(18.3)$ & 301 (15.9) & $153(17.8)$ & 0.1 \\
\hline CKD, n (\%) & $960(17.9)$ & $524(20.1)$ & $128(6.8)$ & $308(35.8)$ & $<0.0001$ \\
\hline Dialysis, n (\%) & $147(2.8)$ & $106(4.1)$ & $3(0.2)$ & $38(4.4)$ & $<0.0001$ \\
\hline Depression, n (\%) & $1461(27.3)$ & $702(27.0)$ & $556(29.4)$ & $203(23.6)$ & 0.006 \\
\hline PTSD, n (\%) & $931(17.4)$ & $434(16.7)$ & $373(19.8)$ & $124(14.4)$ & 0.001 \\
\hline \multicolumn{6}{|l|}{ Presentation, $\mathrm{n}(\%)$} \\
\hline Stable angina & $210(3.9)$ & $112(4.3)$ & $72(3.8)$ & $26(3.0)$ & \multirow[t]{5}{*}{0.001} \\
\hline Positive functional study & $1290(24.3)$ & $611(23.5)$ & $427(22.6)$ & $252(29.3)$ & \\
\hline Ischemic heart disease & $470(8.8)$ & $246(9.5)$ & $152(8.1)$ & $72(8.4)$ & \\
\hline Chest pain & $3242(60.6)$ & 1558 (59.9) & $1191(63.1)$ & $493(57.3)$ & \\
\hline Acute coronary syndrome & $140(2.6)$ & $76(2.9)$ & $47(2.5)$ & $17(2.0)$ & \\
\hline \multicolumn{6}{|l|}{ Postangiography revascularization, $\mathrm{n}(\%)$} \\
\hline None & $2361(44.1)$ & $1160(44.6)$ & $794(42.0)$ & $407(47.3)$ & \multirow[t]{3}{*}{0.02} \\
\hline $\mathrm{PCl}$ & 1763 (32.9) & $878(33.7)$ & $620(32.8)$ & $265(30.8)$ & \\
\hline CABG & 1228 (22.9) & $565(21.7)$ & $475(25.2)$ & $188(21.9)$ & \\
\hline Baseline $\mathrm{HbA} 1 \mathrm{c}(\mathrm{mmol} / \mathrm{mol})$, mean (SD) & $\begin{array}{l}46(4) \\
(6.4 \%(0.6))\end{array}$ & $\begin{array}{l}46(4) \\
(6.4 \%(0.6))\end{array}$ & $\begin{array}{l}48(4) \\
(6.5 \%(0.6))\end{array}$ & $\begin{array}{l}49(4) \\
(6.6 \%(0.6))\end{array}$ & $<0.0001$ \\
\hline \multicolumn{6}{|l|}{ Baseline HbA1c category, $\mathrm{n}(\%)$} \\
\hline$<42 \mathrm{mmol} / \mathrm{mol}(<6 \%)$ & $1055(19.7)$ & $625(24.0)$ & $287(15.2)$ & $143(16.6)$ & \multirow[t]{4}{*}{$<0.0001$} \\
\hline $42-47 \mathrm{mmol} / \mathrm{mol}(6 \%-6.4 \%)$ & $1634(30.5)$ & $846(32.5)$ & $570(30.2)$ & $218(25.4)$ & \\
\hline $48-52 \mathrm{mmol} / \mathrm{mol}(6.5 \%-6.9 \%)$ & $1540(28.8)$ & $692(26.6)$ & $603(31.9)$ & $245(28.5)$ & \\
\hline $53-57 \mathrm{mmol} / \mathrm{mol}(7 \%-7.4 \%)$ & $1123(21.0)$ & $440(16.9)$ & $429(22.7)$ & $254(29.5)$ & \\
\hline Diabetes duration (years), mean (SD) & $3.7(2.71)$ & $3.8(3.0)$ & $3.4(2.4)$ & $3.9(2.4)$ & $<0.0001$ \\
\hline
\end{tabular}

BMI, body mass index; CABG, coronary artery by pass graft; CAD, coronary artery disease; CHF, congestive heart failure; CKD, chronic kidney disease; COPD, chronic obstructive pulmonary disease; $\mathrm{HbA} 1 \mathrm{c}$, hemoglobin $\mathrm{A} 1 \mathrm{c}$; PAD, peripheral arterial disease; PCI, percutaneous coronary intervention; PTSD, post-traumatic stress disorder.

to be treated with just a sulfonylurea at 1 year after catheterization.

The unadjusted 2-year mortality rates were $6.6 \%, 5.2 \%$, and $11.9 \%$ in the no medications, non-sulfonylurea, and sulfonylurea categories, respectively $(\mathrm{p}<0.0001$ for unadjusted between-group differences in mortality rate; table 3 and figure 1A). Among sulfonylurea users, mortality rates differed non-significantly between those taking glyburide and those taking other sulfonylureas (8.8\% and $13.1 \%$ in those taking glyburide and other sulfonylureas, respectively; $\mathrm{p}=0.07$; online supplementary tables 1 and 2). IPW-standardized 2-year mortality 
Table 2 Covariate balance between medication categories before and after inverse probability of treatmentweighted standardization

\begin{tabular}{|c|c|c|}
\hline Covariate & $\begin{array}{l}\text { Maximum between- } \\
\text { group standardized } \\
\text { difference } \\
\text { before weighting (\%) }\end{array}$ & $\begin{array}{l}\text { Maximum between- } \\
\text { group standardized } \\
\text { difference } \\
\text { after weighting (\%) }\end{array}$ \\
\hline Age & 40.6 & 5.4 \\
\hline Male & 10.6 & 7.4 \\
\hline \multicolumn{3}{|l|}{ Race } \\
\hline White & 13.8 & 5.2 \\
\hline Black & 15.6 & 5.7 \\
\hline Asian & 8.1 & 8.1 \\
\hline Pacific Islander & 4.1 & 2.4 \\
\hline Native American & 8.4 & 4.5 \\
\hline $\begin{array}{l}\text { Family history of } \\
C A D\end{array}$ & 11.1 & 2.5 \\
\hline Tobacco & 8.0 & 7.0 \\
\hline Hypertension & 11.6 & 3.4 \\
\hline Hyperlipidemia & 9.0 & 5.0 \\
\hline BMI & 13.2 & 2.2 \\
\hline $\begin{array}{l}\text { Framingham risk } \\
\text { score }\end{array}$ & 19.8 & 5.8 \\
\hline PAD & 15.7 & 5.9 \\
\hline $\mathrm{CHF}$ & 30.8 & 6.1 \\
\hline COPD & 6.2 & 2.5 \\
\hline CKD & 75.7 & 14.7 \\
\hline Dialysis & 26.1 & 14.0 \\
\hline Depression & 13.1 & 2.5 \\
\hline PTSD & 14.1 & 4.3 \\
\hline \multicolumn{3}{|l|}{ Presentation } \\
\hline Stable angina & 6.9 & 7.5 \\
\hline $\begin{array}{l}\text { Positive functional } \\
\text { study }\end{array}$ & 15.7 & 4.2 \\
\hline $\begin{array}{l}\text { Ischemic heart } \\
\text { disease }\end{array}$ & 5.9 & 1.7 \\
\hline Chest pain & 13.8 & 2.0 \\
\hline \multicolumn{3}{|l|}{$\begin{array}{l}\text { Postangiography } \\
\text { revascularization }\end{array}$} \\
\hline None & 10.7 & 4.6 \\
\hline $\mathrm{PCl}$ & 6.2 & 3.5 \\
\hline CABG & 8.0 & 4.2 \\
\hline Baseline HbA1c & 32.4 & 4.8 \\
\hline Diabetes duration & 16.6 & 5.3 \\
\hline
\end{tabular}

$\mathrm{BMI}$, body mass index; CABG, coronary artery bypass graft; CAD, coronary artery disease; $C H F$, congestive heart failure; CKD, chronic kidney disease; COPD, chronic obstructive pulmonary disease; HbA1c, hemoglobin A1c; PAD, peripheral arterial disease; $\mathrm{PCl}$, percutaneous coronary intervention; PTSD, post-traumatic stress disorder.

rates were $5.9 \%$ (95\% CI 5.0 to 6.9 ), $6.5 \%$ (95\% CI 5.1 to 7.9 ), and $9.7 \%$ (95\% CI 7.7 to 11.8 ) in the no medications, non-sulfonylurea, and sulfonylurea categories, respectively $(p<0.0001$ for adjusted between-group differences in mortality rate). The absolute mortality difference between non-sulfonylurea and sulfonylurea groups was $3.2 \%$ (95\% CI 0.7 to 5.7$)$ ( $\mathrm{p}=0.01$; table 4$)$. After IPW standardization, the absolute mortality difference between the no medication and non-sulfonylurea medication categories was non-significant $(-0.6 \%(95 \%$ CI -2.2 to 1.1$), \mathrm{p}=0.5)$.

In secondary analysis, we used the IPW Cox proportional hazards models to estimate relative hazards of 2-year mortality across the different medication categories. In the IPW Cox proportional hazards models, the overall type 3 test for variation in hazard of mortality across all three medication categories was non-significant $(\mathrm{p}=0.07)$. In pairwise comparisons between medication categories, sulfonylurea use showed a non-significant trend toward greater hazard of mortality compared with non-sulfonylurea medication use (HR 1.38 (95\% CI 1.00 to 1.93), $\mathrm{p}=0.05$; table 4 and figure $1 \mathrm{C}$ ), whereas mortality risk was clearly comparable in those on no medications relative to non-sulfonylurea users (HR 1.02 (95\% CI 0.77 to 1.35 ), $\mathrm{p}=0.9$; table 4 and figure $1 \mathrm{C}$ ).

We performed two sensitivity analyses to address possible sources of residual confounding. First, we excluded all participants (a total of 43 individuals) who underwent cardiac catheterization at sites where there were no patients in the sulfonylurea category, and this had no effect on the primary results (online supplementary table 3). Second, we repeated the analyses after excluding patients with CKD or who use dialysis (a total of 964 individuals) as these variables are independent predictors of mortality, are more prevalent among those using sulfonylureas than among those in the other two medication categories, and remained unbalanced across medication categories even in the IPW study population. When excluding patients with CKD or dialysis use, the incidence rate of mortality declined in all three medication categories (table 3), but the pattern of unadjusted association with survival was similar to that observed in the full cohort (figure 1C). Individuals taking non-sulfonylurea medications had the lowest unadjusted mortality rate, followed by those on no diabetes medications, with those taking sulfonylureas having the highest mortality (table 3 and figure 1C). The IPW-standardized 2-year mortality rates after excluding individuals with CKD or on dialysis were $4.7 \%$ (95\% CI 3.7 to 5.6$), 5.5 \%(95 \%$ CI 4.4 to 6.7 ), and $8.4 \%$ (95\% CI 5.9 to 10.9 ) in the no medications, non-sulfonylurea, and sulfonylurea categories, respectively (table 4). The absolute mortality difference between non-sulfonylurea and sulfonylurea groups remained significant (IPW-standardized risk difference of $2.9 \%$ (95\% CI 0.1 to 5.6 ), $\mathrm{p}=0.04$; table 4 ), and the relative risk of mortality associated with sulfonylurea use compared with non-sulfonylurea medication use increased slightly (HR 1.52 (95\% CI 1.04 to 2.21), $\mathrm{p}=0.03$; table 4 ). As in the full cohort, mortality did not differ significantly between individuals on no diabetes medications and those taking a non-sulfonylurea medication (IPW-standardized risk difference of $-0.9(95 \%$ 
Table 3 Crude mortality rates across diabetes medication categories in the full study cohort and after excluding individuals with chronic kidney disease or on dialysis

\begin{tabular}{|c|c|c|c|c|}
\hline & All participants & No medications & $\begin{array}{l}\text { Non-sulfonylurea } \\
\text { users }\end{array}$ & Sulfonylurea users \\
\hline Full cohort & $371 / 5352(6.9 \%)$ & $171 / 2603(6.6 \%)$ & $98 / 1889$ (5.2\%) & $102 / 860(11.9 \%)$ \\
\hline $\begin{array}{l}\text { Excluding participants with } \\
\text { chronic kidney disease or on } \\
\text { dialysis }\end{array}$ & $244 / 4388(5.6 \%)$ & 106/2076 (5.1\%) & $86 / 1760$ (4.9\%) & $52 / 552$ (9.4\%) \\
\hline
\end{tabular}

CI -2.4 to 0.6 ), $\mathrm{p}=0.24$; HR 1.03 (95\% CI 0.76 to 1.38 ), $\mathrm{p}=0.87$; table 4$)$.

\section{DISCUSSION}

In this study, we found that sulfonylurea use was associated with greater 2-year mortality in individuals with well-controlled diabetes and obstructive CAD compared with other oral diabetes medications or no treatment. In fully adjusted survival analysis, mortality differences between sulfonylurea and non-sulfonylurea users did not achieve statistical significance; therefore, mortality differences across medication categories could be attributed to confounding by risk factors for mortality. However, the results of our study suggest caution with sulfonylurea use in individuals with well-controlled diabetes and CAD for two reasons. First, in sensitivity analysis excluding individuals with CKD and dialysis-covariates that remained poorly matched after IPW - the mortality difference between sulfonylurea users and non-sulfonylurea users was statistically significant. Second, the point estimate for the association between sulfonylurea use and mortality, compared with non-sulfonylurea use, was substantial-38\% higher, although the result was not statistically significant owing to wide CIs. We also found that sulfonylurea use was common in our study population, $16 \%$ of all participants and $31 \%$ of those taking medications, consistent with prior studies in non-VA populations, ${ }^{16}{ }^{17}$ suggesting that oral diabetes treatment optimization might impact a substantial proportion of individuals with diabetes and CAD. By limiting the study to individuals with well-controlled diabetes, we conclude that differences in mortality between treatment groups are unlikely to be explained by differences in levels of glycemic control. By focusing on 2-year mortality, our results suggest that active diabetes treatment optimization at the time of MI or cardiac catheterization could impact short-term clinical outcomes, even after HbA1cbased glycemic control goals have been achieved.

As diabetes management guidelines have increasingly focused on individualization of treatment, evidence to support tailoring treatment is needed. By focusing on a well-defined high-risk population of patients with diabetes and $\mathrm{CAD}$, and by examining short-term outcomes, our study complements and extends the findings of prior trials and comparative effectiveness studies of metformin and sulfonylureas for diabetes treatment. ${ }^{8-11} 1424$ Our results support the findings of a small randomized trial that found improved 5-year mortality outcomes associated with metformin treatment compared with sulfonylurea treatment in individuals with diabetes and $\mathrm{CAD}^{24} \mathrm{On}$ the other hand, our results contrast with those of a study of individuals with diabetes hospitalized with acute MI, which showed that individuals treated with sulfonylureas had lower in-hospital mortality than those on no diabetes medications or on regimens that did not include a sulfonylurea. ${ }^{28}$ This difference may be explained by the fact that our study and the prior study of patients with acute MI focused on very different clinical outcomes-2-year mortality versus in-hospital mortality. In addition, most of the individuals in the sulfonylurea-treated group in the prior study were also taking additional diabetes medications, so whether the association with mortality was attributable to sulfonylureas or to a combination treatment approach is unclear. $^{28}$

Both the Action in Diabetes and Vascular Disease: Preterax and Diamicron MR Controlled Evaluation (ADVANCE) $^{29}$ and Thiazolidinediones Or Sulfonylureas and Cardiovascular Accidents.Intervention Trial (TOSCA.IT) ${ }^{30}$ randomized trials evaluated sulfonylureas as add-on therapy to other diabetes medications, and neither study observed higher mortality with sulfonylurea use. Most, if not all, participants in the ADVANCE and TOSCA.IT studies were on multiple diabetes medications. Although gliclazide was the principal study drug employed in those randomized to intensive rather than standard glycemic treatment in ADVANCE, nearly $60 \%$ of participants in the standard glycemic control arm were on a sulfonylurea during follow-up, and most participants were on additional diabetes medications $(70 \%$ on metformin, $24 \%-40 \%$ on insulin). ${ }^{29}$ In TOSCA.IT, individuals with $\mathrm{HbAlc} 53-75 \mathrm{mmol} / \mathrm{mol}$ (7\%-9\%) despite metformin treatment were randomized to receive either pioglitazone or a sulfonylurea; therefore, the primary treatment contrast was between sulfonylureas and pioglitazone. ${ }^{30}$ Thus, the designs of the ADVANCE and TOSCA. IT studies make it difficult to make any inferences about the comparative safety of monotherapy with sulfonylureas and other oral diabetes treatments in individuals with underlying CAD.

The results of our study also contrast with the results of the Bypass Angioplasty Revascularization Investigation 2 Diabetes (BARI-2D) study that compared medical versus revascularization approaches for treatment of $\mathrm{CAD}$, and insulin sensitization (primarily metformin 
A.

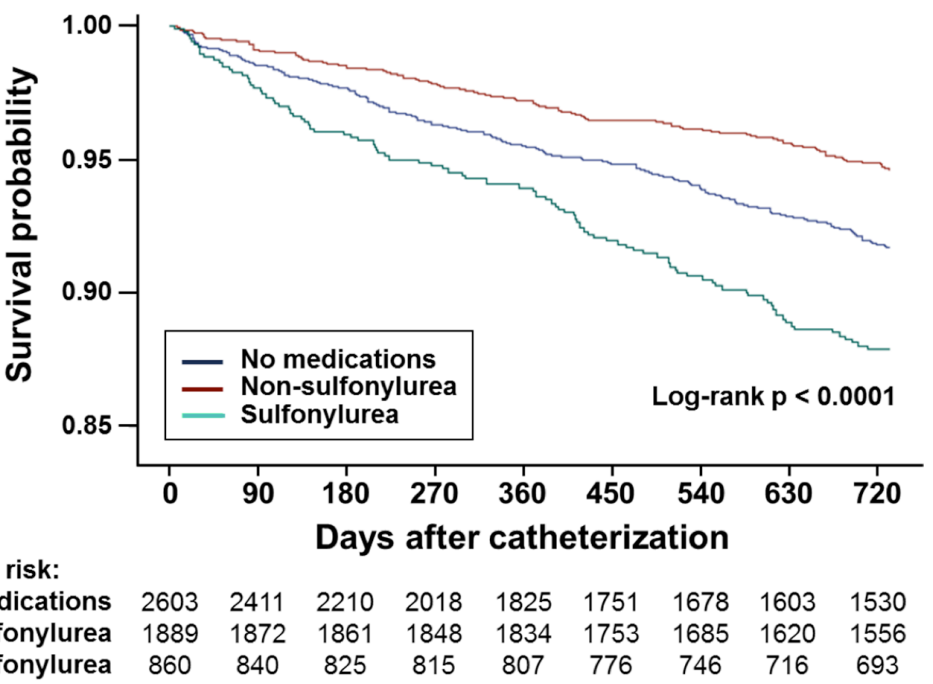

Number at risk:

$\begin{array}{rccccccccc}\text { No medications } & 2603 & 2411 & 2210 & 2018 & 1825 & 1751 & 1678 & 1603 & 1530 \\ \text { Non-sulfonylurea } & 1889 & 1872 & 1861 & 1848 & 1834 & 1753 & 1685 & 1620 & 1556 \\ \text { Sulfonylurea } & 860 & 840 & 825 & 815 & 807 & 776 & 746 & 716 & 693\end{array}$

B.



C.

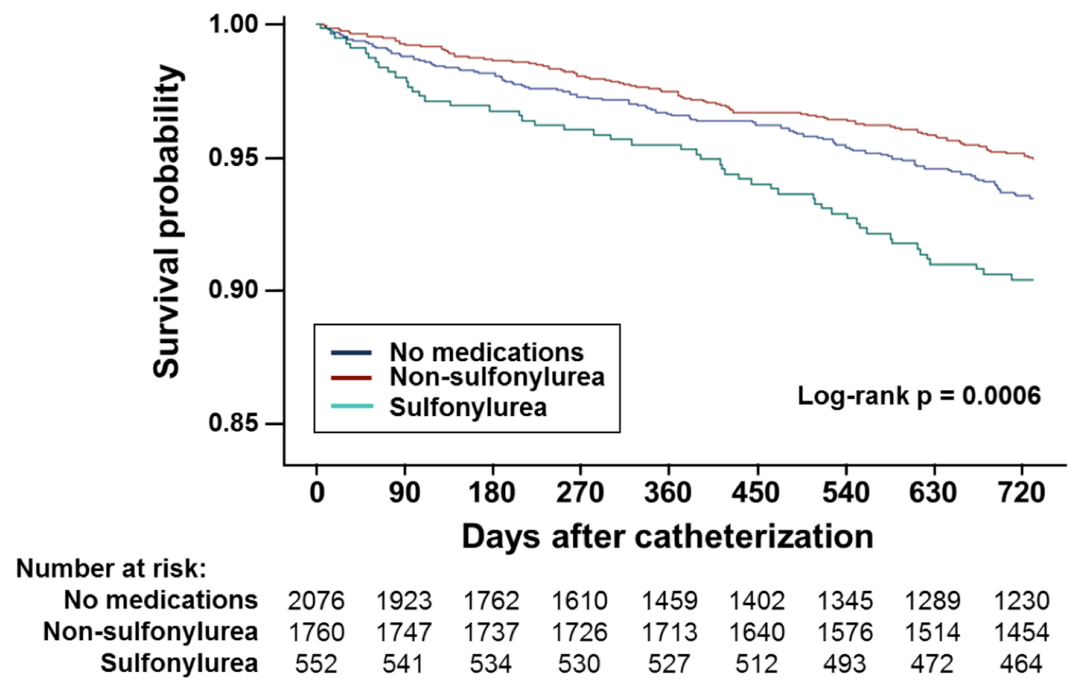

Figure 1 Unadjusted and adjusted survival after cardiac catheterization in individuals on no diabetes medications, taking non-sulfonylurea oral diabetes medication, and sulfonylureas. Unadjusted survival and the number at risk in each medication category are shown for the full study cohort (A); adjusted survival probabilities from multivariable Cox proportional hazards models comparing individuals in each medication category are shown in (B). Unadjusted survival and the number at risk in each medication category after excluding individuals with chronic kidney disease or on dialysis are shown in (C). 
Table 4 Association between baseline diabetes medication category and 2-year mortality

\begin{tabular}{|c|c|c|c|c|c|}
\hline & $\begin{array}{l}\text { Adjusted mortality } \\
\text { rate }^{*}(95 \% \mathrm{Cl})\end{array}$ & $\begin{array}{l}\text { Risk difference } \\
(95 \% \mathrm{Cl})\end{array}$ & $P$ values & $\mathrm{HR}+(95 \% \mathrm{Cl})$ & $P$ values \\
\hline \multicolumn{6}{|l|}{ Full cohort } \\
\hline Non-sulfonylurea & $6.5 \%(5.1$ to 7.9$)$ & Ref & Ref & Ref & Ref \\
\hline Sulfonylurea & $9.7 \%$ (7.7 to 11.8$)$ & $3.2(0.7$ to 5.7$)$ & 0.01 & 1.38 (1.00 to 1.93$)$ & 0.05 \\
\hline No medications & $5.9 \%(5.0$ to 6.9$)$ & $-0.6(-2.2$ to 1.1$)$ & 0.5 & 1.02 (0.77 to 1.35$)$ & 0.9 \\
\hline \multicolumn{6}{|c|}{$\begin{array}{l}\text { Excluding participants with CKD } \\
\text { or on dialysis }\end{array}$} \\
\hline Non-sulfonylurea & $5.5 \%$ (4.4 to 6.7$)$ & Ref & Ref & Ref & Ref \\
\hline Sulfonylurea & $8.4 \%$ (5.9 to 10.9$)$ & $2.9(0.1$ to 5.6$)$ & 0.04 & 1.52 (1.04 to 2.21$)$ & 0.03 \\
\hline No medications & $4.7 \%$ (3.7 to 5.6$)$ & $-0.9(-2.4$ to 0.6$)$ & 0.24 & $1.03(0.76$ to 1.38$)$ & 0.87 \\
\hline
\end{tabular}

${ }^{*}$ All-cause mortality rate in each medication category in the inverse probability of treatment-weighted population.

†HR for 2-year mortality in individuals taking sulfonylureas or no medications compared with those taking non-sulfonylurea medication in inverse probability of treatment-weighted Cox proportional hazards models additionally adjusted for chronic kidney disease and dialysis. CKD, chronic kidney disease ref, reference.

or thiazolidinedione therapy) versus insulin provision (primarily sulfonylurea or insulin therapy) approaches for treatment of diabetes. ${ }^{31}$ BARI-2D demonstrated no difference in all-cause mortality or major adverse cardiovascular events between individuals randomized to insulin sensitization as compared with insulin provision therapy. However, there are several important differences between our study and BARI-2D. First, and most importantly, over $40 \%$ of BARI-2D participants randomized to the insulin sensitization treatment arm were still treated with insulin or sulfonylureas during the study, and the most common treatment in the insulin provision arm was insulin. Therefore, the treatment comparison in BARI-2D diverged substantially from the present study. Second, the study populations differ: BARI-2D participants were younger (62.4 vs 67.1 years of age), had a longer diabetes duration at baseline (10.4 vs 3.7 years), had higher baseline HbA1c (61 mmol/mol (7.7\%) vs 46 $\mathrm{mmol} / \mathrm{mol}(6.4 \%))$, were more likely to be taking insulin at baseline (28\% vs none in our study), and were more likely to have had a prior MI $(32 \%$ in BARI-2D, whereas our study was limited to individuals without a history of CAD). The BARI-2D population likely represented a less healthy population from a cardiometabolic perspective than the population in our VA observational study. Third, we observed a higher overall mortality rate than in BARI2D: $6.9 \%$ 2-year mortality in our study compared with $13.3 \%$ 5-year mortality in BARI-2D. Taken together, the results of our study at minimum suggest caution specifically with sulfonylurea treatment in older individuals with well-controlled glycemia and newly diagnosed $\mathrm{CAD}$, even if the BARI-2D study did not find evidence in support of a broader strategy of insulin sensitization compared with insulin provision in patients with diabetes and CAD. In sum, our study extends the existing literature by focusing on near-term safety and efficacy of oral diabetes treatments in a specific high-risk population, thus providing evidence that may inform guideline-recommended diabetes treatment individualization.

Although we found increased mortality in individuals with CAD treated with sulfonylureas, our study does not elucidate the mechanism by which sulfonylurea use might lead to increased mortality or whether there are drug-specific associations with mortality within the sulfonylurea class. Among sulfonylureas in wide use in contemporary clinical practice, glyburide is associated more strongly with hypoglycemia than glipizide and glimepiride. ${ }^{32}{ }^{33}$ In addition, glyburide, but not glimepiride or glipizide, is believed to limit ischemic preconditioning, suggesting drug-specific cardiac effects. ${ }^{11}{ }^{28}{ }^{34-36}$ In contrast to prior work, two recent studies argue against greater cardiovascular toxicity of glyburide compared with glipizide. One, using a large national cohort in the UK, did not observe a difference in acute MI, ischemic stroke, cardiovascular death, or all-cause mortality when comparing different classes of sulfonylurea medications. ${ }^{37}$ In that study, however, those taking glyburide or glimepiride were at significantly increased risk of hypoglycemia compared with those taking glipizide, gliclazide, or tolbutamide. ${ }^{37}$ A second study specifically evaluated the risk of sudden cardiac death or ventricular arrhythmia in US sulfonylurea users and found that glyburide was associated with a slightly lower risk of sudden cardiac death or ventricular arrhythmia than glipizide. ${ }^{38}$ In our study, the majority of participants (69\%) used glipizide, and we did not observe a significant difference in mortality between glyburide users and those using glipizide or glimepiride. Thus, we conclude from our results that higher short-term mortality among sulfonylurea users compared with those taking non-sulfonylurea diabetes medications is a class effect, rather than a drug-specific effect, although we were unable to distinguish between direct cardiotoxicity, increased frequency and/or severity of hypoglycemia, or alternative explanations. 
Head-to-head studies demonstrating better outcomes with metformin than with sulfonylureas could not distinguish whether metformin has beneficial cardiovascular effects or if sulfonylureas have cardiotoxic affects. ${ }^{8}$ We did not observe significant differences in mortality between individuals treated with non-sulfonylurea medications (predominantly metformin) and those treated with no diabetes medications, but did observe increased mortality with sulfonylurea use. Assessing treatment-related outcomes in individuals with $\mathrm{CAD}$ is of particular importance since there is conflicting evidence regarding the cardiovascular safety of sulfonylureas, and diabetes and CAD co-occur frequently. ${ }^{11} 2324283439-42$ Our study suggests a neutral effect of metformin use and increased harms associated with sulfonylurea treatment in individuals with CAD and well-controlled glycemia, although we cannot determine if this is via a direct effect of sulfonylureas. Finally, by limiting our study population to those with well-controlled diabetes (and including baseline HbAlc and diabetes duration in the treatment propensity model), we conclude that the association between medication category and mortality is independent of glycemic control. This is consistent with the findings of recent cardiovascular outcomes trials with newer diabetes medications that found associations between treatment and clinical outcomes independent of glycemia. ${ }^{3-7}$

Our study has important limitations. As with all observational studies, causal inference is limited. Our IPW approach cannot account for unmeasured factors associated with treatment choice. However, we included many relevant covariates, including comorbidities and other clinical care variables associated with both treatment choice and outcomes, and demonstrated adequate confounder balance in our IPW sample. While we cannot exclude residual confounding, our results were stable in two sensitivity analyses addressing imbalance in CKD/dialysis and variation across VA sites. Second, our study population was predominantly male; our results warrant validation in a cohort with greater representation of women, but there is no a priori reason to expect sex-specific associations between diabetes medications and mortality. Third, we were unable to determine if hypoglycemia was a mediator of the association between sulfonylurea use and mortality. Only severe hypoglycemia can be assessed using the VA electronic health record, but likely underestimates the frequency of clinically important hypoglycemic events. The rates of severe hypoglycemia necessitating clinical care were too low in our data to perform an adequately powered mediation analysis. Finally, by limiting the study population to individuals with well-controlled diabetes and on at most one oral medication, we diminished the generalizability of our results. Doing this, however, permitted better control of confounding and the potential to isolate the association between a single medication and outcomes, increasing internal validity.

Despite the limitations, our study has several clinically relevant implications. First, we did not identify a short-term mortality benefit of metformin treatment in individuals with CAD for whom ongoing diabetes treatment is needed. Second, diabetes medication choice, in addition to personalized glycemic control goals, is an important consideration in optimal individualized diabetes care; in this case, a diagnosis of CAD may warrant re-evaluation of diabetes treatment in individuals with good glycemic control. Third, recent randomized trials suggest that the members of the sodium-glucose co-transporter-2 (SGLT-2) inhibitor and glucagon-like peptide-1 (GLP-1) agonist classes of diabetes medication are associated with improved cardiovascular outcomes in high-risk individuals. ${ }^{3-7}$ However, the vast majority of participants in those studies had long-standing diabetes and were on one or more glycemic therapies to which the study drug (or placebo) was added. ${ }^{3-7}$ Head-to-head data of monotherapy with newer classes of medications compared with metformin and sulfonylureas are currently lacking. Additional evidence addressing the relative safety and efficacy of sulfonylureas compared with other diabetes medications in combination with metformin is expected from the Glycemia Reduction Approaches in Diabetes: A Comparative Effectiveness Study clinical trial, which is focused on identifying optimal secondary treatment for individuals who remain above their glycemic control goals on metformin alone. ${ }^{43}$ Finally, individuals with objectively determined CAD using sulfonylurea monotherapy had higher short-term mortality than those taking non-sulfonylurea medications. Our adjusted results in the full study population did not achieve statistical significance, and causal conclusions are limited in this observational study. However, the magnitude of the association of sulfonylurea use with mortality and the improved statistical significance of the association in sensitivity analysis in a more well-matched study population suggest sulfonylureas should be used with caution in those with CAD and diabetes. In this high-risk population, providers may want to review sulfonylurea use and consider substituting metformin, if tolerated and not contraindicated, or newer diabetes agents with superior cardiovascular safety data.

\section{Author affiliations}

${ }^{1}$ Section of Hospital Medicine, Veterans Affairs Eastern Colorado Healthcare System, Denver, Colorado, USA

${ }^{2}$ Division of General Internal Medicine, University of Colorado School of Medicine, Aurora, Colorado, USA

${ }^{3}$ Colorado Cardiovascular Outcomes Research Consortium, Aurora, Colorado, USA ${ }^{4}$ Division of Endocrinology, University of Colorado School of Medicine, Aurora,

Colorado, USA

${ }^{5}$ Department of Biostatistics and Informatics, Colorado School of Public Health, Aurora, Colorado, USA

${ }^{6}$ Division of Cardiology, Washington University School of Medicine, St Louis, Missouri, USA

${ }^{7}$ Division of General Medicine and Clinical Epidemiology, Department of Medicine, University of North Carolina School of Medicine, Chapel Hill, North Carolina, USA ${ }^{8}$ Division of Rheumatology, University of Colorado School of Medicine, Aurora, Colorado, USA

Contributors SR, WGL, and DRS conceived of and designed the study; WGL and TMM contributed to data acquisition and availability; SR, WGL, and GKG contributed to analysis plan refinement and performed all data analyses. All authors contributed 
to interpretation of the results, as well as to drafting and critical revision of the manuscript. All authors approved of the version submitted for review and publication.

Funding SR is supported by a University of Colorado School of Medicine, Division of General Internal Medicine Small Grant and American Heart Association Award 17MCPRP33670728. DRS was supported by the VA Advanced Fellowship Program in HSR\&D. LC is supported by VA HSR\&D IRR 14-048-3. SAB is supported by the National Institute of Diabetes and Digestive and Kidney Diseases of the National Institutes of Health under Award Number K23DK109200. The content is solely the responsibility of the authors and does not necessarily represent the official views of the National Institutes of Health or the Department of Veterans Affairs.

Competing interests None declared.

Patient consent Not required.

Ethics approval The local VA Research and Development Committee and the Colorado Multiple Institutional Review Board provided approval for this study. Provenance and peer review Not commissioned; externally peer reviewed. Data sharing statement A limited dataset without identifiable participant data is available on request.

Open access This is an Open Access article distributed in accordance with the Creative Commons Attribution Non Commercial (CC BY-NC 4.0) license, which permits others to distribute, remix, adapt, build upon this work non-commercially, and license their derivative works on different terms, provided the original work is properly cited and the use is non-commercial. See: http://creativecommons.org/ licenses/by-nc/4.0/

(C) Article author(s) (or their employer(s) unless otherwise stated in the text of the article) 2018. All rights reserved. No commercial use is permitted unless otherwise expressly granted.

\section{REFERENCES}

1. Haffner SM, Lehto S, Rönnemaa T, et al. Mortality from coronary heart disease in subjects with type 2 diabetes and in nondiabetic subjects with and without prior myocardial infarction. N Engl J Med 1998;339:229-34.

2. Tseng CL, Soroka O, Maney M, et al. Assessing potential glycemic overtreatment in persons at hypoglycemic risk. JAMA Intern Med 2014;174:259-68.

3. Marso SP, Daniels GH, et al. Liraglutide and cardiovascular outcomes in type 2 diabetes. N Engl J Med 2016;375:311-22.

4. Zinman B, Wanner C, Lachin JM, et al. Empagliflozin, cardiovascular outcomes, and mortality in type 2 diabetes. N Engl J Med 2015;373:2117-28.

5. Marso SP, Bain SC, Consoli A, et al. Semaglutide and cardiovascular outcomes in patients with type 2 diabetes. $N$ Engl J Med 2016;375:1834-44.

6. Neal B, Perkovic V, Mahaffey KW, et al. Canagliflozin and cardiovascular and renal events in type 2 diabetes. $N$ Engl J Med 2017;377:644-57.

7. Holman RR, Bethel MA, Mentz RJ, et al. Effects of once-weekly exenatide on cardiovascular outcomes in type 2 diabetes. $N$ Engl J Med 2017;377:1228-39.

8. Azoulay L, Suissa S. Sulfonylureas and the Risks of Cardiovascular Events and Death: A Methodological Meta-Regression Analysis of the Observational Studies. Diabetes Care 2017;40:706-14.

9. Roumie CL, Hung AM, Greevy RA, et al. Comparative effectiveness of sulfonylurea and metformin monotherapy on cardiovascular events in type 2 diabetes mellitus: a cohort study. Ann Intern Med 2012;157:601-10.

10. Wheeler S, Moore K, Forsberg CW, et al. Mortality among veterans with type 2 diabetes initiating metformin, sulfonylurea or rosiglitazone monotherapy. Diabetologia 2013;56:1934-43.

11. Schramm TK, Gislason GH, Vaag A, et al. Mortality and cardiovascular risk associated with different insulin secretagogues compared with metformin in type 2 diabetes, with or without a previous myocardial infarction: a nationwide study. Eur Heart $J$ 2011;32:1900-8

12. Monami M, Genovese S, Mannucci E. Cardiovascular safety of sulfonylureas: a meta-analysis of randomized clinical trials. Diabetes Obes Metab 2013;15:938-53.

13. Monami M, Marchionni N, Masotti G, et al. Effect of combined secretagogue/biguanide treatment on mortality in type 2 diabetic patients with and without ischemic heart disease. Int $J$ Cardiol 2008;126:247-51.
14. Phung OJ, Schwartzman E, Allen RW, et al. Sulphonylureas and risk of cardiovascular disease: systematic review and meta-analysis. Diabet Med 2013;30:1160-71.

15. Varvaki Rados D, Catani Pinto L, Reck Remonti L, et al. The association between sulfonylurea use and all-cause and cardiovascular mortality: a meta-analysis with trial sequential analysis of randomized clinical trials. PLoS Med 2016;13:e1001992.

16. Berkowitz SA, Krumme AA, Avorn J, et al. Initial choice of oral glucose-lowering medication for diabetes mellitus: a patientcentered comparative effectiveness study. JAMA Intern Med 2014;174:1955-62

17. Hampp C, Borders-Hemphill V, Moeny DG, et al. Use of antidiabetic drugs in the U.S., 2003-2012. Diabetes Care 2014;37:1367-74.

18. American Diabetes Association. 8. Pharmacologic Approaches to Glycemic Treatment. Diabetes Care 2017;40(Suppl 1):S64-S74.

19. American Diabetes Association. 6. Glycemic targets. Diabetes Care 2017;40(Suppl 1):S48-56.

20. Hernández AV, Boersma E, Murray GD, et al. Subgroup analyses in therapeutic cardiovascular clinical trials: are most of them misleading? Am Heart J 2006;151:257-64.

21. Sun X, Briel M, Busse JW, et al. Credibility of claims of subgroup effects in randomised controlled trials: systematic review. BMJ 2012;344:e1553.

22. Wallach JD, Sullivan PG, Trepanowski JF, et al. Evaluation of evidence of statistical support and corroboration of subgroup claims in randomized clinical trials. JAMA Intern Med 2017;177:554-60.

23. Garratt KN, Brady PA, Hassinger NL, et al. Sulfonylurea drugs increase early mortality in patients with diabetes mellitus after direct angioplasty for acute myocardial infarction. J Am Coll Cardiol 1999;33:119-24.

24. Hong J, Zhang Y, Lai S, et al. Effects of metformin versus glipizide on cardiovascular outcomes in patients with type 2 diabetes and coronary artery disease. Diabetes Care 2013;36:1304-11.

25. Maddox TM, Plomondon ME, Petrich $M$, et al. A national clinical quality program for Veterans Affairs catheterization laboratories (from the Veterans Affairs clinical assessment, reporting, and tracking program). Am J Cardiol 2014;114:1750-7.

26. Miller DR, Safford MM, Pogach LM. Who has diabetes? Best estimates of diabetes prevalence in the department of veterans affairs based on computerized patient data. Diabetes Care 2004;27(Suppl 2):B10-21

27. McCaffrey DF, Griffin BA, Almirall D, et al. A tutorial on propensity score estimation for multiple treatments using generalized boosted models. Stat Med 2013;32:3388-414.

28. Zeller M, Danchin N, Simon D, et al. Impact of type of preadmission sulfonylureas on mortality and cardiovascular outcomes in diabetic patients with acute myocardial infarction. J Clin Endocrinol Metab 2010;95:4993-5002.

29. Patel A, MacMahon S, Chalmers J, et al. Intensive blood glucose control and vascular outcomes in patients with type 2 diabetes. $N$ Engl J Med 2008;358:2560-72.

30. Vaccaro O, Masulli M, Nicolucci A, et al. Effects on the incidence of cardiovascular events of the addition of pioglitazone versus sulfonylureas in patients with type 2 diabetes inadequately controlled with metformin (TOSCA.IT): a randomised, multicentre trial. Lancet Diabetes Endocrinol 2017;5:887-97.

31. Frye RL, August P, Brooks MM, et al. A randomized trial of therapies for type 2 diabetes and coronary artery disease. $N$ Engl J Med 2009;360:2503-15.

32. Holstein A, Plaschke A, Egberts EH. Lower incidence of severe hypoglycaemia in patients with type 2 diabetes treated with glimepiride versus glibenclamide. Diabetes Metab Res Rev 2001;17:467-73.

33. van Staa T, Abenhaim L, Monette J. Rates of hypoglycemia in users of sulfonylureas. J Clin Epidemiol 1997;50:735-41.

34. Lee TM, Chou TF. Impairment of myocardial protection in type 2 diabetic patients. J Clin Endocrinol Metab 2003;88:531-7.

35. Lee TM, Su SF, Chou TF, et al. Loss of preconditioning by attenuated activation of myocardial ATP-sensitive potassium channels in elderly patients undergoing coronary angioplasty. Circulation 2002; 105:334-40.

36. Rezende PC, Rahmi RM, Hueb W. The influence of diabetes mellitus in myocardial ischemic preconditioning. $J$ Diabetes Res 2016;2016:8963403-.

37. Douros A, Yin $\mathrm{H}, \mathrm{Yu} \mathrm{OHY}$, , et al. Pharmacologic differences of sulfonylureas and the risk of adverse cardiovascular and hypoglycemic events. Diabetes Care 2017;40:1506-13.

38. Leonard CE, Brensinger CM, Aquilante CL, et al. Comparative safety of sulfonylureas and the risk of sudden cardiac arrest and ventricular arrhythmia. Diabetes Care 2018;41:dc170294-. 
39. Emdin CA, Klarin D, Natarajan P, et al. Genetic variation at the sulfonylurea receptor, type 2 diabetes, and coronary heart disease. Diabetes 2017;66:2310-5.

40. Middleton TL, Wong J, Molyneaux L, et al. Cardiac effects of sulfonylurea-related hypoglycemia. Diabetes Care 2017;40:663-70.

41. Scognamiglio R, Negut C, Ramondo A, et al. Detection of coronary artery disease in asymptomatic patients with type 2 diabetes mellitus. J Am Coll Cardiol 2006;47:65-71.
42. Pladevall M, Riera-Guardia N, Margulis AV, et al. Cardiovascular risk associated with the use of glitazones, metformin and sufonylureas: meta-analysis of published observational studies. BMC Cardiovasc Disord 2016;16:14

43. Nathan DM, Buse JB, Kahn SE, et al. Rationale and design of the glycemia reduction approaches in diabetes: a comparative effectiveness study (GRADE). Diabetes Care 2013;36:2254-61. 